CrossMark \& click for updates

Cite this: Mater. Horiz., 2016, 3, 478

Received 4th August 2016,

Accepted 12th September 2016

DOI: $10.1039 / c 6 m h 00289 g$

www.rsc.li/materials-horizons

\title{
Annihilation upconversion in nanoconfinement: solving the oxygen quenching problem
}

\begin{abstract}
Stanislav Baluschev, ${ }^{\star a b}$ Kartheek Katta, ${ }^{a}$ Yuri Avlasevich ${ }^{a}$ and Katharina Landfester*a
This article reviews different approaches towards the protection of optically excited organic triplet ensembles in nanoconfinement against quenching and photoinduced oxidation by molecular oxygen. We compare and discuss passive and active protection. The passive protection is based on the application of high-barrier materials for the encapsulation of the active substances which prevent oxygen molecules from penetration and physical contact with the excited states, and active protection is based on the application of sacrificial singlet oxygen scavenging species. The long-term performance of different protective techniques together with the efficiency of the annihilation upconversion process in nanoconfinement are outlined.
\end{abstract}

\section{Introduction}

Triplet excited state chromophores are being applied in various fields including bioimaging, ${ }^{1}$ molecular sensing, ${ }^{2}$ and photocatalytic organic reactions. ${ }^{3}$ One of the most promising applications based on excited triplet ensembles is the photon energy upconversion by triplet-triplet annihilation (TTA-UC). Recently the TTA-UC

${ }^{a}$ Max Planck Institute for Polymer Research, Ackermannweg 10, D-55128 Mainz, Germany. E-mail: landfest@mpip-mainz.mpg.de

${ }^{b}$ Optics and Spectroscopy Department, Faculty of Physics,

Sofia University "St. Kliment Ochridski”, 5 James Bourchier, 1164 Sofia, Bulgaria. E-mail: balouche@phys.uni-sofia.bg

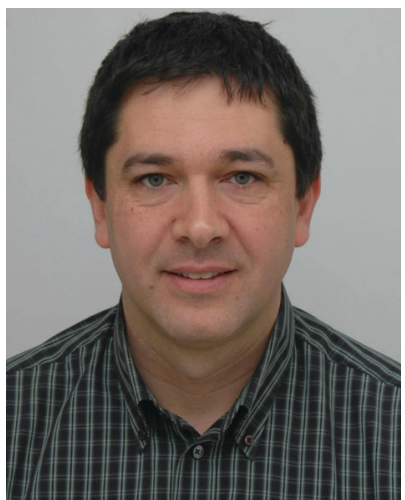

Stanislav Baluschev
Stanislav Baluschev studied Laser Physics at the Sofia University "Saint Kliment Ochridski", Bulgaria, where he received his PhD. In 1996, he was granted a DAAD- research fellowship at Hannover University, Germany; in 1999 - a Feinberg Research Fellowship at the Department of Complex Systems, Weizmann Institute of Science, Israel; during the academic year 2014/2015 he was a FRIAS-fellow, University of Freiburg, Germany. In 2001, he joined the group of Prof. Gerhard Wegner/Prof. Katharina Landfester at MPI for Polymer Research. His current research interests include nonlinear optics, atomic physics and annihilation upconversion in organic systems. process was demonstrated using ultralow intensity, noncoherent excitation light (less than 1-4 $\mathrm{mW} \mathrm{cm}^{-2}$ ), corresponding to the solar intensity at the Earth's surface. ${ }^{4}$ Such a low-excitation threshold allows for the development of several unique applications in materials science, ${ }^{5}$ solar cell devices, ${ }^{6}$ solar fuels, ${ }^{7}$ bioimaging, ${ }^{8}$ and for the extension of the infrared limit of oxygenic photosynthesis. ${ }^{9}$

The TTA-UC process takes place in multi-chromophore systems consisting of energetically optimized pairs of sensitizer (metallated macrocycles) and emitter molecules (aromatic hydrocarbons), as shown in Fig. 1. The photon energy is absorbed by a sensitizer and stored in its triplet state (Fig. 1, the light green arrows (plain and dotted) represent the absorption of two excitation photons), formed in the process of intersystem crossing (ISC).

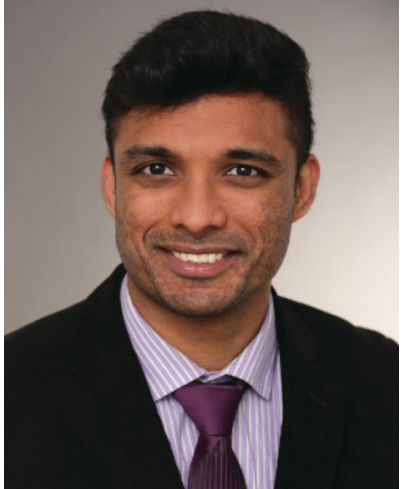

Kartheek Katta
Kartheek Katta received his Master's degree from the University of Siegen, Germany, in December 2012. He is currently pursuing a dual PhD degree in Chemistry from the University of Mainz and the Seoul National University with work being conducted at the Max Planck Institute for Polymer Research and Seoul National University since May 2013. His research is focused on luminescence properties in polymer and polymer/ inorganic nanocapsules. 


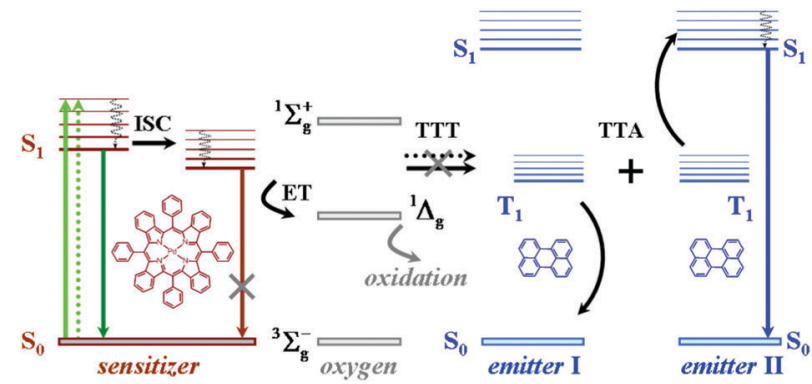

Fig. 1 General energetic scheme of triplet-triplet annihilation upconversion in an oxygen contaminated environment.

Further, this energy is transferred in two successive time moments (the black arrows (plane and dotted), marked with TTT) to an emitter triplet state via the process of triplet-triplet transfer (TTT). Next, the excited triplet states of the two emitter molecules undergo triplet-triplet annihilation (TTA), in which one emitter molecule returns back to its singlet ground state and the other molecule gains the energy of both triplet states and is excited to the higher singlet state. As the singlet state emitter decays radiatively back to the ground state, a delayed fluorescence photon (the blue arrow, Fig. 1) bearing a higher energy than that of the excitation photons is emitted. It is important to note that all of the levels in the TTA-UC process involving energetic levels are real levels, and indeed no virtual energy levels are used. The efficiency of the process of TTT towards the excited triplet states of the emitter ensemble depends on the degree of overlap of the triplet manifolds of the sensitizer and emitter. Therefore, almost all TTA-UC systems represent, alongside the delayed emitter fluorescence

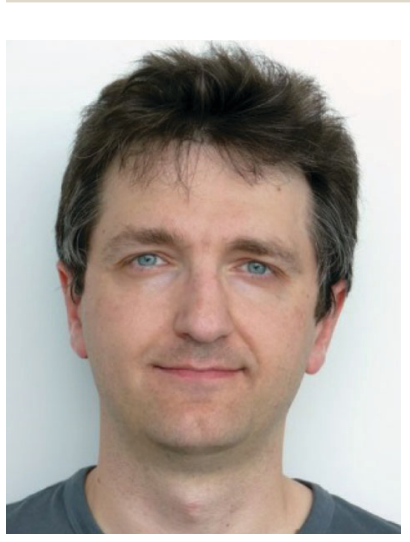

Yuri Avlasevich
Yuri Avlasevich received his $\mathrm{MSci}$ in organic chemistry in 1993 from the Belarusian State University, and his $P h D$ in polymer chemistry in 2000 from the Institute of Physical Organic Chemistry, National Academy of Sciences (both in Minsk, Belarus). After being a visiting scientist at the Institute of Physical Chemistry in Warsaw, Poland, and at the Institute of General Botany, University of Mainz, he joined the Synthetic Chemistry Group at Max Planck Institute for Polymer Research in 2003. In 2009 he moved to the Physical Chemistry of Polymers Group, where he is working now as a research chemist in the field of upconverting dyes. (the UC-signal, Fig. 1, the blue arrow), the residual sensitizer phosphorescence (Fig. 1, the brown arrow).

The above-described excitation energy transfers occur in an oxygen-free environment. In an environment, contaminated with molecular oxygen even at the ppm-level, the TTA-UC process becomes more complicated. Besides the processes of TTT, TTA, and subsequent emitter fluorescence, the residual sensitizer phosphorescence will also be substantially affected: the energy stored in the excited states of the triplet ensembles is being actively dissipated competing with emissive (phosphorescence) or non-emissive (triplet to triplet) energy transfer processes. The reason for this is the process of energy transfer between the triplet state of the organic molecule and the ground state of molecular oxygen, leading to the generation of singlet oxygen $\left({ }^{1} \Delta_{\mathrm{g}}\right.$, Fig. 1). Singlet oxygen is a highly reactive species, leading to oxidation of the photoactive molecules, followed by further loss of efficiency.

Thus, to fully exploit the TTA-UC process in different applications, the development of an effective protection strategy against quenching by molecular oxygen and protection against the subsequent production of highly reactive singlet oxygen is essential. In bulk TTA-UC samples, the oxygen/active dye molar concentration ratio is at the level of $10: 1$, in contrast with the case of nanoconfined materials this ratio is at least $200: 1$. Such a relative quencher excess will affect the optical response of the studied nano-sized UC-sample, causing pronounced oxygen quenching of a delayed emitter fluorescence signal and modifying the residual sensitizer phosphorescence. Simultaneously, effective photo-oxidation of the used organic dyes will be observed. This effect could be an additional source of uncertainty when the fluorescence signal is used as a quantitative measure in the field of life-science sensing. ${ }^{10}$

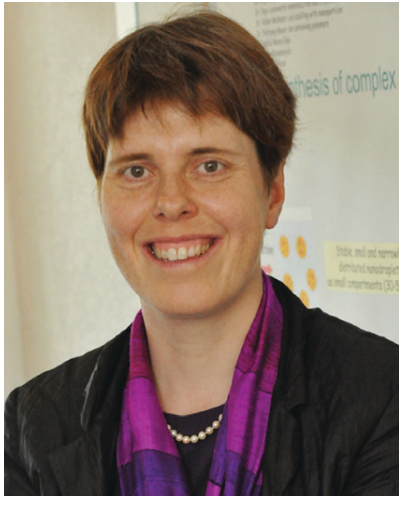

Katharina Landfester
Katharina Landfester joined the Max Planck Society in 2008 as one of the directors of the MPI for Polymer Research. She studied Chemistry at the Technical University of Darmstadt and in Strasbourg. In 1995, she received her doctoral degree in Physical Chemistry after working with Prof. H. W. Spiess at the MPIP. In 1996, she moved for a doctoral stay at the Lehigh University and in 1998 at the MPI of Colloids and Interfaces in Golm. In 2003, she accepted a chair (C4) of Macromolecular Chemistry at the University of Ulm. In 1992 and 1994, and 1996 she obtained stipends for her research activities in Strasbourg and in the US. In 1998, she received the Liebig stipend of the Chemical Industry Fund (FCI). In 2001 she was awarded the Reimund Stadler prize of the German Chemical Society (GDCh) and the prize of the Dr Hermann Schnell Foundation, followed by the Bruno Werdelmann Lecturer in 2012 and the BAYER Lecturer in 2014. 


\section{Results and discussion}

The research studies presented in this mini-review are illustrated in Fig. 2. In particular, two main strategies, leading to oxygen protection of the excited triplet states can be considered: (1) passive protection, based on the application of barrier materials for sealing, packaging or encapsulation of the active substances, which prevent oxygen molecules from penetration and physical contact with the excited states; and (2) active protection, based on the application of oxygen scavenging species which react with singlet oxygen to minimize the amount of oxygen available for deteriorative reactions, leading to chemical degradation of the photoactive molecules.

Having in mind the targeted application - using the TTA-UC process in a nanoconfined environment, the straight forward solution to the oxygen problem - sealing of the UC-sample under glove-box conditions does not appear as an applicable technology. Passive protection includes (i) armouring of the nanocontainer shell with crystalline materials with low oxygen permeability (Fig. 2, the blue spheres); (ii) encapsulation into nano- and microcarriers using shell material polymers with low oxygen permeability or (iii) incorporation of crystalline materials into the nanocontainer shell (Fig. 2, the black rectangles), simultaneously optimizing the shell-materials towards reducing the free volume. Active protection includes (iv) the addition of sacrificial oxygen scavengers into the hydrophobic core or at the hydrophilic phase of the nanocontainer suspension, (v) chemical decoration of the used sensitizer molecules with specially designed singlet oxygen traps (Fig. 2, decorated with anthracene substituents: palladium porphyrin) and (vi) enrichment or complete exchange of the core-matrix of the hydrophobic nanocontainer with a solvent, demonstrating singlet oxygen scavenging properties (Fig. 2, organophosphates with terminal double bonds). All these techniques have particular advantages, therefore implementation of a specific protection methodology depends on the envisaged application. The materials further elucidate step by step the necessary synthetic changes

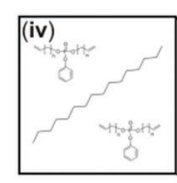

(iii)
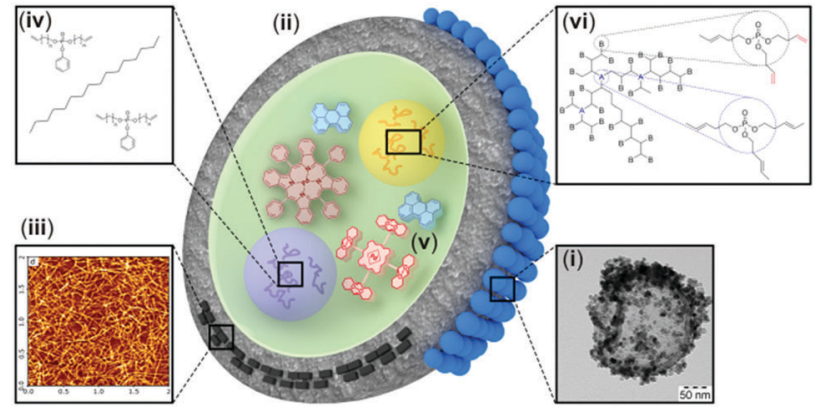

Fig. 2 Schematic representation of the protective strategies: (i) TEM of a NC armoured with cerium oxide; (ii) polymer shell with a low oxygen permeability; (iii) incorporation of crystalline materials into the nanocontainer shell and the AFM image of TEMPO-NFC adsorbed on top of mica; ${ }^{14}$ (iv) addition of sacrificial oxygen scavengers into the hydrophobic core; (v) chemical decoration of the sensitizer molecules with singlet oxygen traps; ${ }^{17}$ and (vi) exchange of the core-matrix with a singlet oxygen scavenging solvent. ${ }^{18}$ leading to long-term protection of the TTA-UC process against oxygen quenching.

(i) The oxygen protective technique, which changes the nanocontainer architecture as little as possible is represented by the armouring of the nanocontainer shell with crystalline materials with low oxygen permeability (Fig. 2, the blue spheres). In particular the armouring introduces an additional property to an already created nanocontainer with specific characteristics. A scanning electron microscopy (SEM) image (Fig. 3c) of hybrid nanocapsules armoured by cerium oxide and a transmission electron microscopy (TEM) image (Fig. 3d) of the same batch of nanocapsules demonstrate that cerium oxide is crystallized on the surface. It is important to notice that neither the nanocontainer-core (loaded materials and matrix/solvent), nor the nanocontainer shell is changed. For instance, cerium oxide nanocrystals can reduce the amount of penetrating oxygen, and so can reduce the photo-induced damage on the nanocontainercore materials (Fig. 3). Despite this protective technique being less invasive, the oxygen permeation decrease has a moderate impact.

The TTA-UC dyes were encapsulated within a rigid silica shell using a self-assembly microemulsion process. ${ }^{19}$ The nanocapsules form a homogeneous suspension that can produce intense, diffuse UC emission in water without deoxygenation, additionally the silica shell provides sites for further surface modification (Fig. 4).

(ii) The idea of including the interacting excited-state species into nanometer-scale shells is quite general and easily adaptable for a large number of small molecules exploited in sensitized upconversion and other photonic applications. For instance, using the microfluidic technique combined with photoinduced free-radical interfacial polymerization of trimethylolpropane
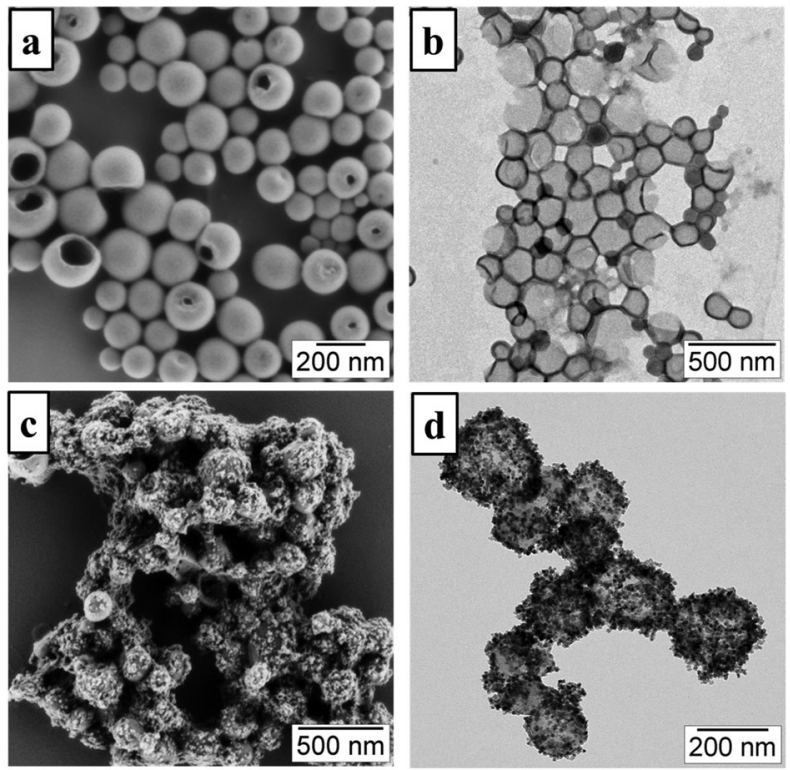

Fig. 3 Microscopic images of carboxylate functionalized polystyrene hybrid nanocapsules containing terrylene diimide: (a) SEM and (b) TEM; (c) SEM image and (d) TEM image of the formation of cerium oxide on the surface of carboxylate functionalized polystyrene nanocapsules. 


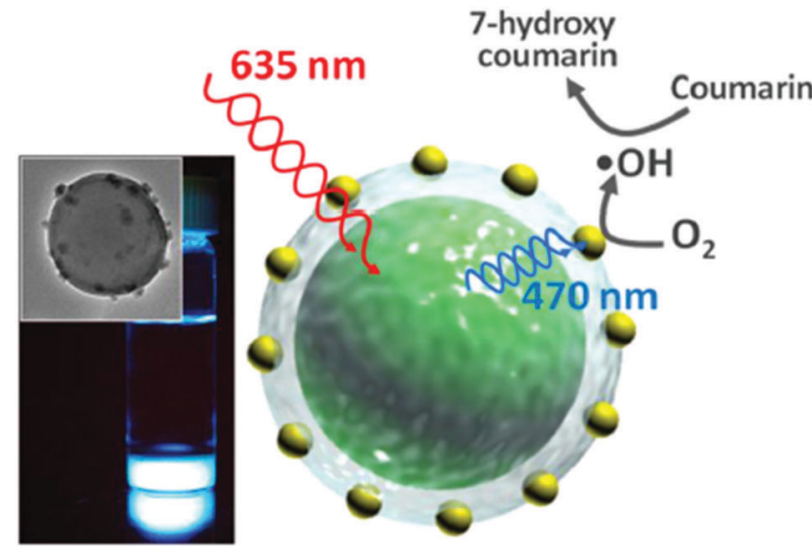

Fig. 4 Aqueous stable nano-scale encapsulation of TTA-UC. Copyright: American Chemical Society.

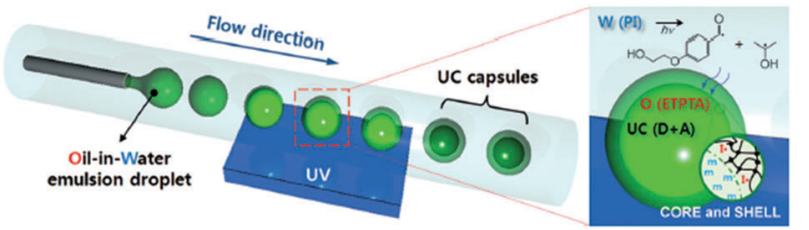

Fig. 5 Fabrication of monodisperse capsules for TTA-UC through interfacial free radical photo-polymerization; (UV = ultraviolet curing excitation, $\mathrm{W}=$ water, $\mathrm{O}=$ oil, $\mathrm{PI}=$ photo-initiator, $\mathrm{m}=$ monomer, $\mathrm{IC}=$ photo-initiator radical, $\mathrm{D}=$ sensitizer, and $\mathrm{A}=$ emitter). ${ }^{11}$ Copyright: Wiley- $\mathrm{VCH}$ Verlag $\mathrm{GmbH}$.

triacrylate (ETPTA) led to capsules, consisting of a fluidic active core (loaded with the UC-active dyes) and an elastomeric shell for mechanical integrity and passive oxygen protection (Fig. 5). ${ }^{11}$ The characteristics of the capsules, particularly the size and shell thickness, can be controlled through the UV exposure time. ETPTA encapsulation retards the penetration of oxygen and the liquid-phase core provides highly efficient UC emission.

Despite the good long-term stability reported by this encapsulation technique, significant uncertainty of the UC-stability is caused by the necessity to apply UV-curing light, which excites simultaneously the sensitizer molecules in their Soret-band: as a result, even at very low oxygen contamination during the preparation procedure, the UC-materials can be photo-oxidized. ${ }^{12}$

Passive protection against oxygen quenching for TTAupconversion was observed in the aqueous phase by encapsulating the solvent phase containing a benchmark platinum(II) octaethylporphyrin-diphenylanthracene sensitizer-acceptor pair in a rigid polymer shell in the form of aqueous dispersible microcapsules. ${ }^{20}$ A mixture of hexadecane and polyisobutylene was used as the inner solvent phase. Such a solvent system enables stable TTA-UC to occur even after a month of exposure to the ambient environment.

Microcapsules that achieve multicolor TTA-UC in both aqueous and dry phases without a deoxygenating-step were prepared ${ }^{21}$ with PtTPBP as a sensitizer and perylene, bis(phenylethynyl)anthracene and a boron dipyrromethene derivative as acceptors for red to blue, cyan, and green UC, respectively (Fig. 6).
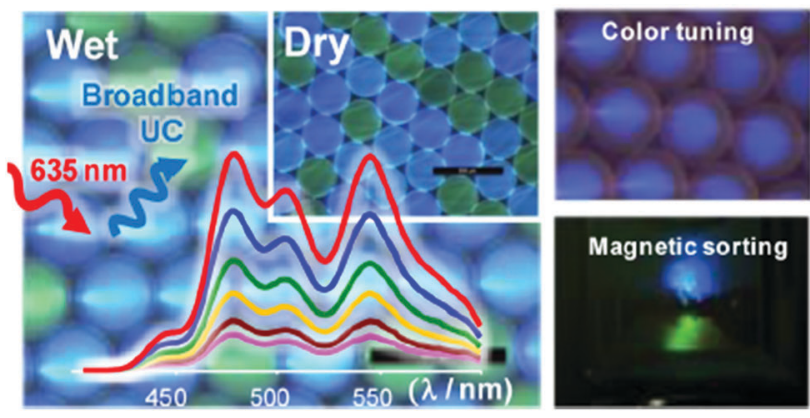

Fig. 6 Upconverting microcapsules for aqueous and dry-phase color tuning and magnetic sorting. Copyright: American Chemical Society.

The nanofiber mats are prepared by simultaneously electrospinning an aqueous solution of a polymer with pronounced oxygen-barrier properties and functional nanocapsules containing a sensitizer/emitter couple optimized for TTA-UC. The optical functionality of the nanocapsules is preserved during the electrospinning process (Fig. 7). The pronounced oxygen-barrier properties of the polymer matrix may efficiently prevent the oxygen penetration so that upconversion fluorescence is registered in an ambient atmosphere. ${ }^{22}$

Organogels, formed by ordered self-assembled molecules and stabilized by developed hydrogen bond networks, enable efficient protection of TTA-UC dyes against molecular oxygen ${ }^{23}$ (see Fig. 8).

A matrix-free upconverting system is designed using branched alkyl chains for decoration of both dyes, ${ }^{24}$ the sensitizer and the emitter. A rationally designed triplet sensitizer (modified Pt(II) porphyrin) is homogeneously doped in an energy harvesting liquid emitter with a diphenylanthracene unit (Fig. 9). In this system passive protection against oxygen is achieved by high dye concentrations and low gas permeability of the system itself.

A non-ionic surfactant (polyoxyethanyltocopheryl sebacate) was used for micelle formation, ${ }^{25}$ loaded with the TTA-UC dyes. The obtained micellar structures show very efficient UC emission in a water environment under extremely low excitation light intensity, down to $10 \mathrm{~mW} \mathrm{~cm}^{-2}$.

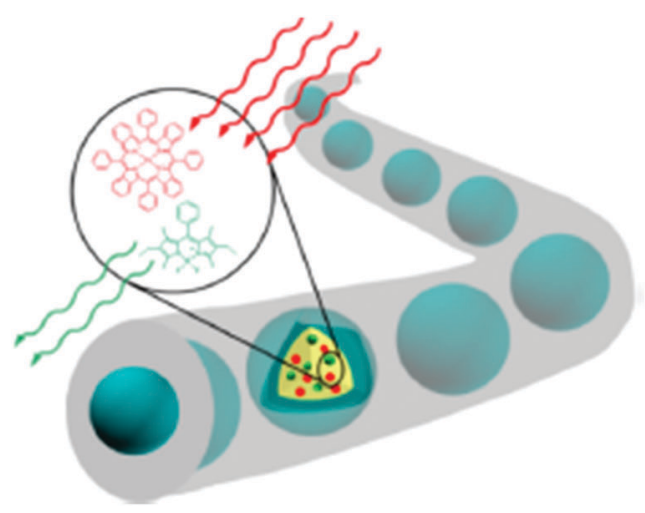

Fig. 7 Schematics of the colloid-electrospinning process for embedding of the TTA-UC nanocapsules in nanofibers. Copyright: American Chemical Society. 


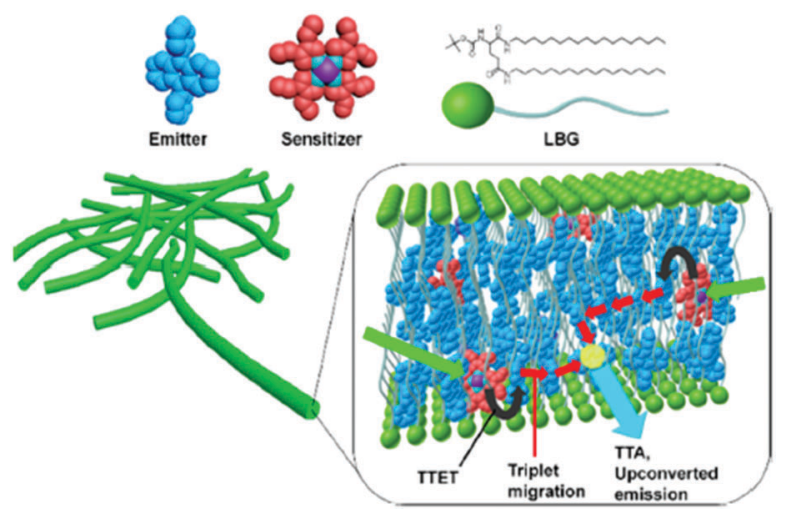

Fig. 8 A schematic representation of the unit structure of the upconversion gel system. Copyright: American Chemical Society.

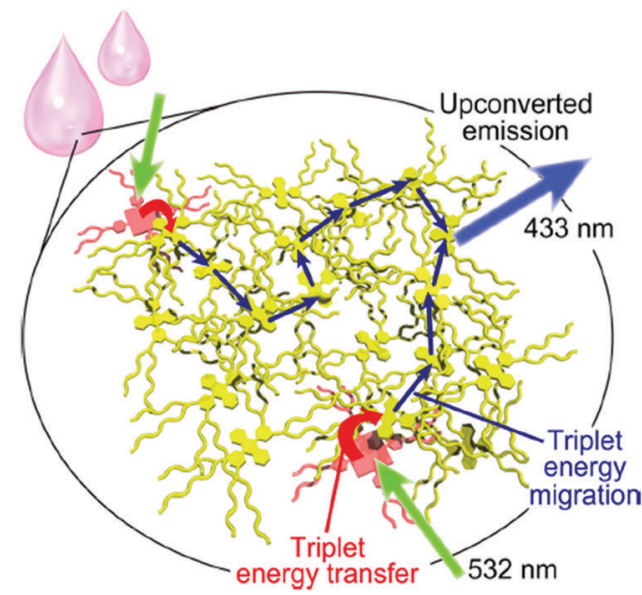

Fig. 9 A schematic representation of the matrix-free liquid TTA-UC system. Copyright: American Chemical Society.

A similar self-assembly strategy was used ${ }^{26}$ for building liposomes capable of generating photons of blue light in situ by triplet-triplet annihilation upconversion of either green or red light. The red-to-blue upconverting liposomes were capable of triggering the photodissociation of ruthenium polypyridyl complexes from PEGylated liposomes using a clinical grade photodynamic therapy laser source (Fig. 10).

(iii) For optical applications, only transparent or semitransparent nanocomposite materials, consisting of amorphous polymer and crystalline nanoparticles with barrier properties can be considered. ${ }^{13}$ In such nanocomposite materials, the solubility of the permeating gas decreases due to the loss of polymer volume available for sorption. Additionally, the increased diffusion path length leads to a loss in diffusivity, i.e., the crystalline nanoparticles act as obstacles. For a successful application of this technique it is important that the particle-surface interaction with the surrounding polymer chains is strong, lowering the mobility of the polymer near the particle surface. It was demonstrated ${ }^{14}$ that cellulose nanofibers (NFCs, Fig. 11) can be regarded as one of the most promising bio-derived materials due to their extraordinary set of physical properties, such as outstanding mechanical properties, optical transparency,

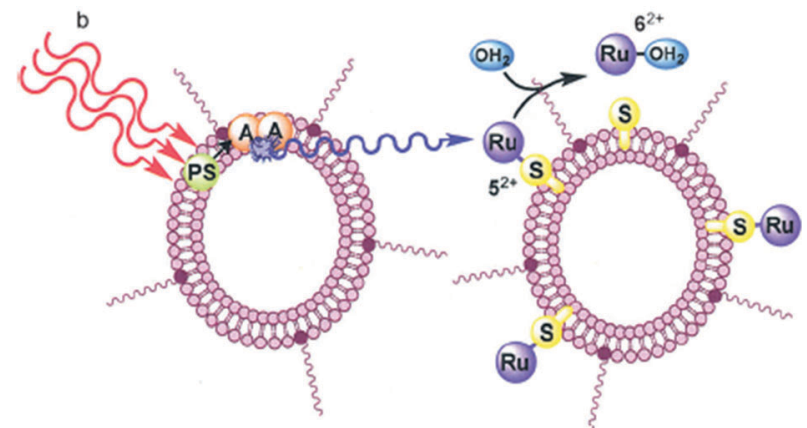

Fig. 10 The TTA-UC process in the lipid bilayer, using a photosensitizer (PS) and an annihilator (A). Copyright: IOP Science.
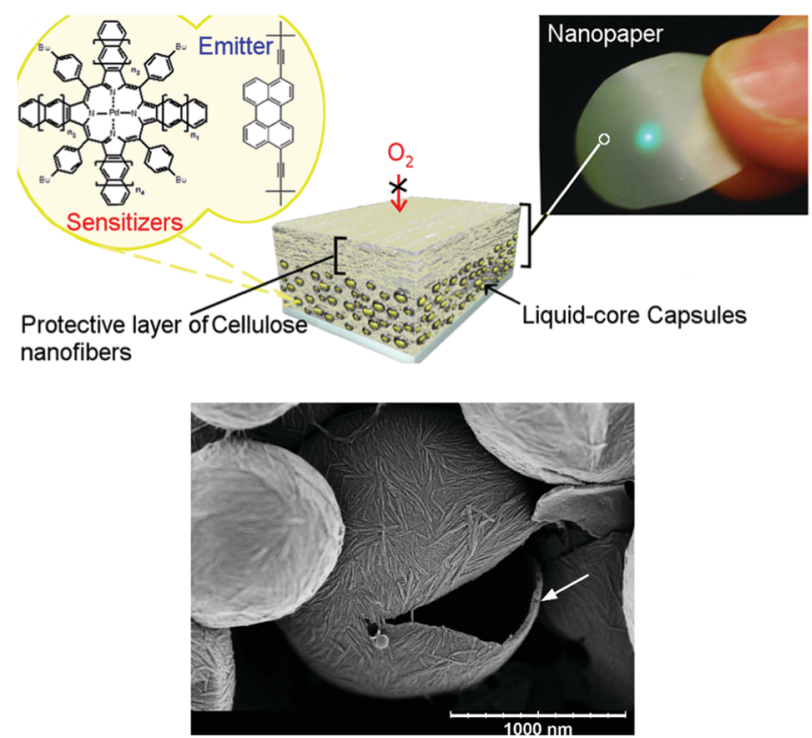

Fig. 11 (Top) Chemical structures of the encapsulated sensitizer family and emitter - 3,9(10)-bis(3,3-dimethylbutyn-1yl)perylene. (Bottom) SEM image of the broken NFC/CNC based capsule. Copyright: VCH Verlag GmbH.

nontoxicity, and ultrahigh oxygen-barrier properties. The oxygenbarrier properties of pristine NFC films are about 2-3 orders of magnitude better than the best petrochemically derived polymer, ethylene vinyl alcohol (at $\left.23{ }^{\circ} \mathrm{C}, 0 \% \mathrm{RH}\right)$. The high barrier properties are a consequence of the semicrystalline structure and optimal packing of nanocellulose in pristine films.

Nanocellulose-based nanocontainers have a liquid core, containing UC-active chromophores and the shell was compounded with NFCs. Due to decreased oxygen permeability and high local mobility of the chromophores, such NFC nanocontainers demonstrate efficient TTA-UC with excellent longterm stability in an oxygen-saturated environment. The lifetime of the UC-process can be further modulated and increased by introducing thicker protective cellulose nanofiber coatings.

Passive protection techniques (i-iii) ensure moderate protection of the optically excited organic triplet ensembles against photo-induced damage caused by the generated singlet oxygen: even if oxygen permeability is reduced by several orders of magnitude, a substantial chemical degradation of active-substances 
is observed. Especially when the nano-confined phosphorescent moieties $^{2}$ or the TTA-UC system is used as a sensing tool, ${ }^{15}$ sustainable and reproducible irradiation of the probing (sensing) emissions - delayed emitter fluorescence (UC-signal) and the residual sensitizer phosphorescence - is obligatory.

(iv) It was demonstrated ${ }^{16}$ that sacrificial oxygen scavengers added at the hydrophilic phase of the nanocontainer suspension allow for effective TTA-UC emission, working at confocal excitation geometry. In this experiment, valinomycin was applied as an oxygen concentration regulator. Valinomycin is an ionophore that has been used to increase the oxygen consumption by the mitochondria of HeLa cells, Fig. 12. Thereby, a decrease of the local oxygen concentration inside the cells is achieved after treatment for $10 \mathrm{~min}$ with valinomycin $\left(1 \mathrm{mmol} \mathrm{L}^{-1}\right)$.

An important disadvantage of this technique is that the total concentration of oxygen (in the ground or in the excited state) is on purpose lowered, therefore a significant part of the dynamic interaction of the excited triplet ensembles with the environment cannot be studied. Further insight to this interaction can be obtained when chemical decoration of the sensitizer molecules, with specially designed singlet oxygen traps, is introduced.

A combination of active and passive protection was demonstrated: ${ }^{27}$ upconversion TTA-UC nanocapsules were developed by loading both the sensitizer and emitter into BSA-dextran covered soybean oil droplets (Fig. 13). This method represents both passive protection by the capsule shell and active protection by the oxygen-scavenging nature of the soybean oil.

Hybrid organic-inorganic systems demonstrated effective energy transfer from the inorganic nanocrystals towards the triplet state of the organic molecules. ${ }^{28}$ Thus, CdSe and PbSe semiconductor nanocrystals are combined with molecular

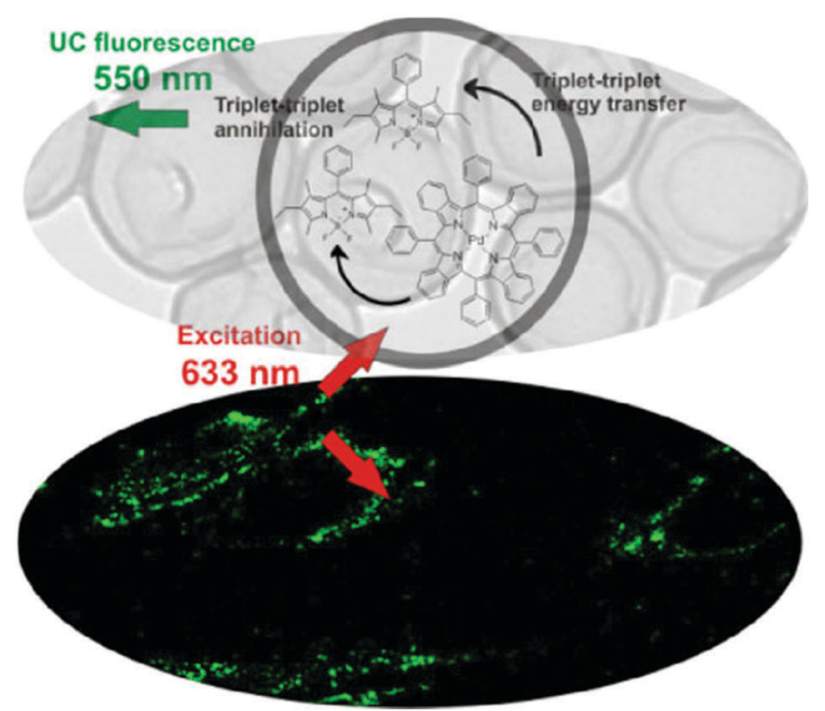

Fig. 12 (Top) TEM image of the TTA-UC nanocontainers (shell - polystyrene-co-polyacrylic acid, core - hexadecane), together with the structures of the sensitizer - palladium(॥) meso-tetraaryltetrabenzoporphyrin and emitter - 1,3,5,7-tetramethyl-8-phenyl-2,6-diethyl dipyrromethane. ${ }^{16}$ (Bottom) CLSM images of living HeLa-cells, incubated with TTA-UC nanocontainers, after $10 \mathrm{~min}$ treatment with valinomycin. Copyright: Wiley-VCH Verlag GmbH \& Co. KGaA.

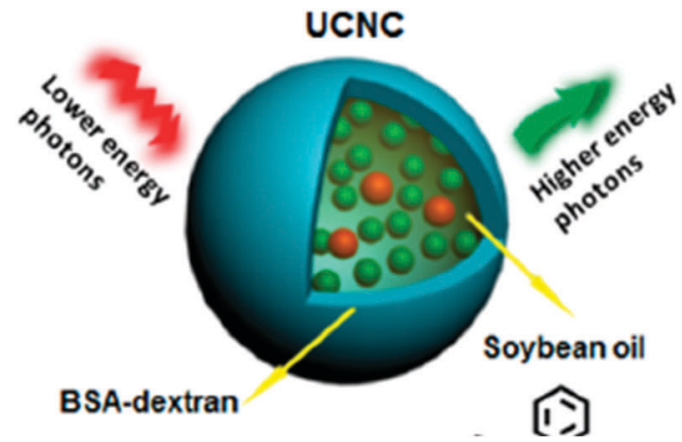

Fig. 13 Schematic representation of the combination of active and passive protection. Copyright: American Chemical Society.

emitters (diphenylanthracene and rubrene) to upconvert photons in both the visible and the near-infrared spectral regions, Fig. 14.

The passive protection scheme, based on metal-organic frameworks (MOF), was used for the creation of organized scaffolds for the UC dyes. ${ }^{29}$ They enable efficient triplet energy migration, annihilation and upconverted singlet energy collection (TEMUPCON). The porous structure of the acceptor-based MOF crystals allows triplet donor molecules to be accommodated without aggregation (Fig. 15).

(v) The synthesis of palladium(II) porphyrin, bearing substituted anthracenes in the meso-positions, which is capable of photoinduced singlet oxygen addition was demonstrated. ${ }^{17}$ Anthracene groups preserve porphyrin phosphorescent properties even in an oxygen rich environment, see Fig. 16.

Furthermore, molecules are provided with structural subunits especially designed to bind molecular oxygen and to not affect the chromophore's properties, allowing the corresponding triplet sensitizer to act in an undisturbed manner. However, upon moderate heating the decorated sensitizer molecule can be fully regenerated - i.e. the oxygen addition is reversible, Fig. 16. Furthermore, protective groups do not bind oxygen in its ground (triplet) state and the corresponding material is stable towards

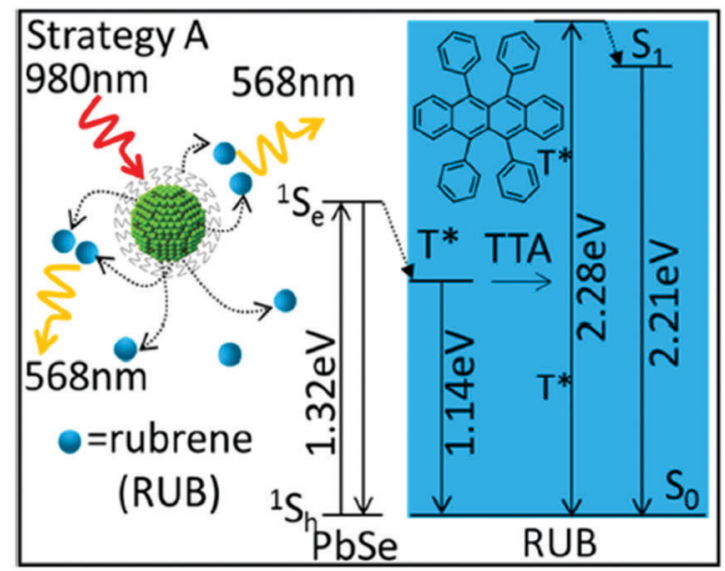

Fig. 14 Schematic illustration of the TTA-UC using a nano-crystal as a sensitizer: the organic ligands of the PbSe NC are electronically inert, and the energy must be transferred directly to the triplet state of the organic emitter RUB. Copyright: American Chemical Society. 


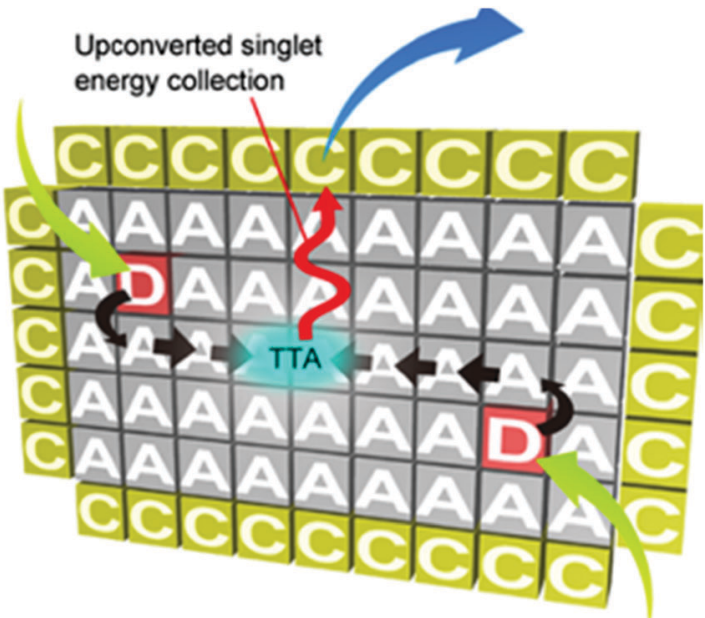

Fig. 15 Scheme for the TTA-UC mechanism based on triplet energy migration, annihilation, and upconverted singlet energy collection (TEM-UPCON). Copyright: American Chemical Society.
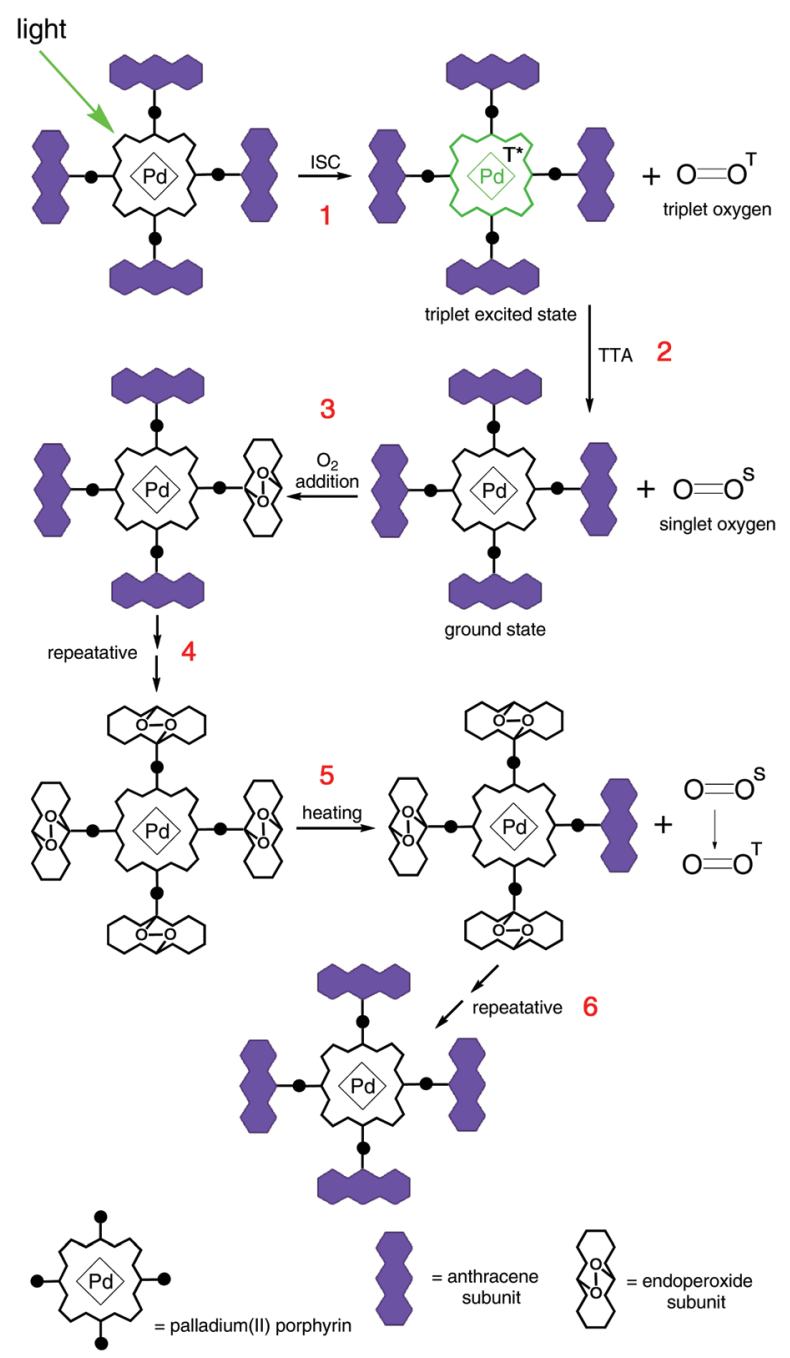

Fig. 16 General scheme of oxygen addition to the triplet sensitizer and further thermal release. Copyright: Royal Society of Chemistry.

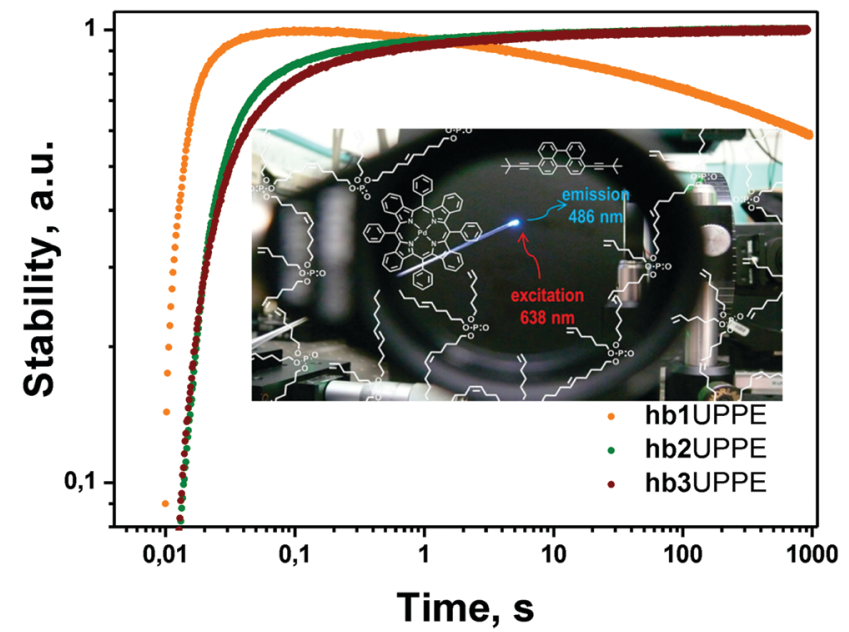

Fig. 17 Long-term stability ${ }^{18}$ of the integral UC fluorescence of the TTA-UC system in different hyperbranched poly(phosphoester)s - hb 1,2,3 under an ambient atmosphere $\left(21 \% \mathrm{O}_{2}\right)$ for continuous irradiation with an excitation intensity of $100 \mathrm{~mW} \mathrm{~cm}^{-2}$. (Inset) Schematic representation of palladium tetrabenzoporphyrin (sensitizer) and 3,9(10)-bis(3,3-dimethylbut-1-yn1-yl)perylene (emitter) in a hyperbranched poly(phosphoester) matrix. Background image: a photograph of a working UC-sample under an ambient environment. Copyright: American Chemical Society.

photooxidation in the course of synthetic procedures and purification. The protection is active only against singlet oxygen, thus only when sensitizer triplet states are formed. Therefore, the presence of an additional stimulus, i.e. optical excitation of the sensitizer, triggers the protection process. This protection strategy is of a sacrificial character, and therefore it is time-limited. The duration of the time span of effective singlet oxygen protection depends on the integral photon flux applied to the sample and on the concentration of the dissolved at the hydrophobic phase oxygen. Having in mind the standard sensitizer concentration for the TTA-UC experiments (at the level of $1 \times 10^{-4} \mathrm{M}$ ), such an active protection strategy can be used for oxygen concentrations in the order of 50-100 ppm for the hydrophobic phase.

(vi) The most promising results were obtained when the corematerial of the hydrophobic nanocontainer was exchanged with a solvent, demonstrating efficient singlet oxygen scavenging properties (Fig. 17); as such, a hyperbranched poly(phosphoester) matrix (hbUPPE) with a high concentration of terminal double bonds was applied. ${ }^{18}$

The protection mechanism is based on the ability of singlet oxygen to react with olefinic double bonds. The particular reaction depends on the nature of the double bonds as different types of addition reactions (epoxides, dioxetanes, alcohols, etc.). The hbUPPEs inherently combine both active and passive protection strategies: namely, passive protection as a consequence of low oxygen permeability and active protection by chemical scavenging of the existing singlet oxygen while being rather inert against triplet oxygen.

\section{Conclusions}

To summarize, in this review article we have tried to shed light on the step by step concepts of annihilation upconversion 
in nanoconfinement. We demonstrate the entanglement of two organic-optoelectronic technologies, namely triplet-triplet annihilation upconversion in a soft-matter environment and miniemulsion polymerization. This enables the combination of the passive and active protection strategies against singlet oxygen quenching and further damaging of the active dyes in densely populated organic triplet ensembles. Miniemulsion polymerization allows for a wide range adjustment of the oxygen permeability of the NC-polymer shell via tuning of its amorphous/crystalline parts and merging with optically stimulated sacrificial singlet oxygen scavenging procedures. For photonic applications, a vital merging of the passive and active type protection in a single nanoconfined object would be optimal, without losing the optical efficiency of the organic ensembles.

\section{Acknowledgements}

K. K. thanks the International Research Training Group 1404 for financial support. S. B. acknowledges the DFNI E 02/11 SunStore-project "Molecular solar thermal systems, enhanced by annihilation upconversion" of the Bulgarian National Science Fund for the financial support. Y. A. acknowledges the project 732794 HypoSens (Horizon 2020 Framework Programme ICT-2016) for the financial support.

\section{Notes and references}

1 J. A. Spencer, F. Ferraro, E. Roussakis, A. Klein, J. Wu, J. M. Runnels, W. Zaher, L. J. Mortensen, C. Alt, R. 1. Turcotte, R. Yusuf, D. Cote, S. A. Vinogradov, D. T. Scadden and C. P. Lin, Nature, 2014, 508, 269-273.

2 D. B. Papkovsky and R. I. Dmitriev, Chem. Soc. Rev., 2013, 42, 8700-8732; M. I. J. Lorenz, S. H. Fischer and O. S. Wolfbeis, Chem. Soc. Rev., 2010, 39, 3102-3114.

3 S. Tombe, E. Antunes and T. Nyokong, J. Mol. Catal. A: Chem., 2013, 371, 125-134.

4 S. Baluschev, T. Miteva, V. Yakutkin, G. Nelles, A. Yasuda and G. Wegner, Phys. Rev. Lett., 2006, 97, 143903; R. R. Islangulov, J. Lott, C. Weder and F. N. Castellano, J. Am. Chem. Soc., 2007, 129(42), 12652-12653.

5 T. F. Schulze, J. Czolk, Y. Y. Cheng, B. Fuckel, R. W. MacQueen, T. Khoury, M. J. Crossley, B. Stannowski, K. Lips and U. Lemmer, J. Phys. Chem. C, 2012, 116, 22794-22801.

6 W. Q. Zou, C. Visser, J. A. Maduro, M. S. Pshenichnikov and J. C. Hummelen, Nat. Photonics, 2012, 6, 560-564.

7 K. Borjesson, D. Dzebo, B. Albinsson and K. Moth-Poulsen, J. Mater. Chem. A, 2013, 1, 8521-8524.

8 J. H. Kim and J. H. Kim, J. Am. Chem. Soc., 2012, 134, 17478; Q. Liu, T. S. Yang, W. Feng and F. Y. Li, J. Am. Chem. Soc., 2012, 134, 5390-5397; C. Wohnhaas, A. Turshatov, V. Mailander, S. Lorenz, S. Baluschev, T. Miteva and K. Landfester, Macromol. Biosci., 2011, 11(6), 772-778; S. M. Borisov, R. Saf, R. Fischer and I. Klimant, Inorg. Chem., 2013, 52(3), 1206-1216; C. Wohnhaas, V. Mailander,
M. Droge, M. A. Filatov, D. Busko, Y. Avlasevich, S. Baluschev, T. Miteva, K. Landfester and A. Turshatov, Macromol. Biosci., 2013, 13, 1422-1430.

9 M. Filatov, S. Ritz, I. Ilieva, V. Mailander, K. Landfester and S. Baluschev, SPIE Newsroom. DOI: 10.1117/2.1201403. 005378. Published Online: April 7, 2014. http://spie.org/ x106642.xml.

10 Z. Gu, L. Yan, G. Tian, S. Li, Z. Chai and Y. Zhao, Adv. Mater., 2013, 25, 3758-3779; C. Hung, W. Huang, Y. Lin, T. Yu, H. Chen, S. Lin, W. Chiang and H. Chiu, Theranostics, 2016, 6, 302-317.

11 H. Kang and E. Reichmanis, Angew. Chem., Int. Ed., 2012, 51, 11841-11844.

12 K. Katta, D. Busko, Y. Avlasevich, R. Muñoz-Espí, S. Baluschev and K. Landfester, Macromol. Rapid Commun., 2015, 36, 1084-1088.

13 S. Hess, M. Demir, V. Yakutkin, S. Baluschev and G. Wegner, Macromol. Rapid Commun., 2009, 30, 394-401.

14 A. J. Svagan, D. Busko, Y. Avlasevich, G. Glasser, S. Baluschev and K. Landfester, ACS Nano, 2014, 8, 8198-8207; A. J. Svagan, C. Bender Koch, M. S. Hedenqvist, F. Nilsson, G. Glasser, S. Baluschev and M. L. Andersen, Carbohydr. Polym., 2016, 136, 292-299.

15 A. Turshatov and S. Baluschev, in Handbook of CoherentDomain Optical Methods, ed. V. V. Tuchin, Springer Science + Business Media, New York, 2013, ISBN-13: 978-1461451754.

16 C. Wohnhaas, V. Mailänder, M. Dröge, M. A. Filatov, D. Busko, Y. Avlasevich, S. Baluschev, T. Miteva, K. Landfester and A. Turshatov, Macromol. Biosci., 2013, 13, 1422-1430.

17 M. A. Filatov, E. Heinrich, D. Busko, I. Z. Ilieva, K. Landfester and S. Baluschev, Phys. Chem. Chem. Phys., 2015, 17, 6501-6510.

18 F. Marsico, A. Turshatov, R. Peköz, Y. Avlasevich, M. Wagner, K. Weber, D. Donadio, K. Landfester, S. Baluschev and F. R Wurm, J. Am. Chem. Soc., 2014, 136, 11057-11064.

19 O. S. Kwon, J.-H. Kim, J. K. Cho and J.-H. Kim, ACS Appl. Mater. Interfaces, 2014, 7, 318-325.

20 J.-H. Kim and J.-H. Kim, J. Am. Chem. Soc., 2012, 134, 17478-17481.

21 J.-H. Kim, F. Deng, F. N. Castellano and J.-H. Kim, ACS Photonics, 2014, 1, 382-388.

22 C. Wohnhaas, K. Friedemann, D. Busko, K. Landfester, S. Baluschev, D. Crespy and A. Turshatov, ACS Macro Lett., 2013, 2, 446-450.

23 P. Duan, N. Yanai, H. Nagatomi and N. Kimizuka, J. Am. Chem. Soc., 2015, 137, 1887-1894.

24 P. Duan, N. Yanai and N. Kimizuka, J. Am. Chem. Soc., 2013, 135, 19056-19059.

25 A. Turshatov, D. Busko, S. Baluschev, T. Miteva and K. Landfester, New J. Phys., 2011, 13, 083035.

26 S. H. C. Askes, A. Bahreman and S. Bonnet, Angew. Chem., Int. Ed., 2014, 53(4), 1029-1033.

27 Q. Liu, B. Yin, T. Yang, Y. Yang, Z. Shen, P. Yao and F. Li, J. Am. Chem. Soc., 2013, 135, 5029-5037. 
28 M. Tabachnyk, B. Ehrler, S. Gelinas, M. L. Bohm, B. J. Walker, K. P. Musselman, N. C. Greenham and R. H. Friendand A. Rao, Nat. Mater., 2014, 13, 1033-1038; Z. Huang, X. Li, M. Mahboub, K. M. Hanson, V. M. Nichols, H. Le, M. L. Tang and C. J. Bardeen, Nano Lett., 2015, 15, 5552-5557; C. Mongin, S. Garakyaraghi, N. Razgoniaeva,
M. Zamkov and F. N. Castellano, Science, 2016, 351(6271), 369-372.

29 P. Mahato, N. Yanai, M. Sindoro, S. Granick and N. Kimizuka, J. Am. Chem. Soc., 2016, 138, 6541-6549; P. Mahato, A. Monguzzi, N. Yanai, T. Yamada and N. Kimizuka, Nat. Mater., 2015, 14, 924-930. 